\title{
La política de distribución comercial en la Europa 2020
}

\author{
Evangelina Aranda García \\ Universidad de Castilla-La Mancha \\ Víctor Jesús Martín Cerdeño \\ Javier Casares Ripol \\ Universidad Complutense de Madrid
}

\section{Resumen}

La Comisión Europea establece que «los sectores comerciales minorista y mayorista están llamados a desempeñar un papel esencial en el fomento del crecimiento y la creación de empleo dentro de la Estrategia Europa 2020: forman parte de los sectores fundamentales que pueden impulsar la transición a una economía y a unos modelos de consumo más sostenibles. La eficiencia en este sector tiene repercusiones sobre la competencia, la innovación, las tendencias de los precios y la competitividad». El artículo pretende poner de manifiesto cómo las actuaciones públicas desarrolladas en el sector comercial, dentro de un "enfoque fines-medios», resultan significativas en el contexto de la Europa 2020. En el trabajo se prestará una atención especial a elementos vinculados con el mercado de trabajo y con el mercado de bienes y servicios.

Palabras clave: distribución comercial, política económica, Unión Europea .

Clasificación JEL: L81, K23, O52.

\begin{abstract}
The European Commission establishes that «the retail and wholesale sectors have an essential role to play in stimulating growth and job creation under the Europe 2020 strategy: they are among the key sectors that can drive the transition to both a more sustainable economy and consumption patterns. Efficiency in this sector has implications for competition, innovation, price trends and competitiveness". The objective of this paper is to show how public performances developed in the commercial sector within an «objetives-instruments approach» prove to be significant in the context of the Europe 2020 strategy. In this work, particular attention is paid to those elements that are linked to the labour market and to the goods and services market.
\end{abstract}

Keywords: retail, economic policy, European Union.

JEL classification: L81, K23, O52.

\section{Introducción}

El sector comercial desempeña un papel esencial en el fomento del crecimiento y la creación de empleo, forma parte de las actividades fundamentales que impulsan 
la transición hacia modelos de consumo más sostenibles. Los objetivos del comercio, materializados en la actividad de las empresas comerciales, son la generación de empleo estable, la contribución a la distribución de la riqueza, la vertebración de la ciudad, la dinamización urbana y el favorecimiento del crecimiento sostenible (Aranda y otros, 2016). La eficiencia en este sector tiene repercusiones sobre la competencia, la innovación, las tendencias de los precios y la competitividad (Comisión Europea, 2013).

En Europa, la consolidación de grandes grupos de distribución ha venido acompañada de un incremento de la concentración empresarial, así como de diversos procesos de integración vertical, creación de centrales de compras, estrategias de diferenciación de enseñas, creación de servicios de valor añadido por los distribuidores y desarrollo de las marcas de distribuidor. Esta situación ha modificado sensiblemente las relaciones entre todos los operadores que participan en la cadena de valor.

La Estrategia Europea 2020 establece cinco objetivos cuantificables para 2020, que marcarán la pauta del proceso y se traducirán en objetivos nacionales: el empleo, la investigación y la innovación, el cambio climático y la energía, la educación y la lucha contra la pobreza. Para que los resultados sean óptimos, la Unión Europea se ha dotado de una nueva gobernanza económica (Semestre Europeo, Pacto de Estabilidad y Crecimiento...), donde se combinan informes generales junto a recomendaciones específicas para cada Estado miembro; en todos ellos se hacen referencia a la necesidad de actuaciones por parte de los decisores públicos en materias relacionadas con finanzas públicas y sistemas de protección social, sector financiero, mercado de trabajo, mercados de bienes y servicios, educación, inclusión social y administración.

Este artículo, en el entorno fines-medios de la política económica, se ha estructurado en dos grandes apartados. El primero de ellos se ha centrado en el análisis de la contribución del comercio a los objetivos de la Estrategia Europea 2020, dejando el segundo para profundizar en las actuaciones públicas llevadas a cabo desde la Unión Europea para la consecución de los mismos. El trabajo termina con un apartado de conclusiones y las principales referencias bibliográficas.

\section{La distribución comercial y los objetivos de la Europa 2020}

El sector distributivo europeo comprende 5,5 millones de empresas, emplea a 29 millones de personas y representa el 11 por 100 del PIB de la Unión Europea (Comisión Europea, 2015). Además, este sector económico participa en la generación de valor añadido por una cantidad cercana al billón de euros (10 por 100 del total de la Unión Europea) junto a otros sectores como la agricultura, la industria agroalimentaria, las tecnologías de la información y la comunicación, el transporte o el sistema financiero. 
Las funciones y labores que realizan los operadores comerciales en la intermediación entre la producción y los consumidores están basadas en la necesidad de salvar las separaciones existentes entre la oferta económica y la demanda final. En este contexto, el papel de las empresas comerciales ha sido básico durante décadas encargándose de salvar la brecha entre producción y consumo que se concreta en torno al espacio, al tiempo y a las diferencias en surtidos o cantidades (Casares y otros, 2013).

Por tanto, la distribución comercial ha pasado de ser un sector reflejo de la producción y del consumo a ser uno de los principales sectores receptores-impulsores de la actividad económica nacional. Las empresas comerciales han generado considerables enlaces hacia delante y hacia atrás y el aparato distributivo ha alcanzado la capitanía de los canales para posibilitar a los productores llegar con sus bienes al consumidor final.

Durante las dos últimas décadas, los importantes cambios que ha experimentado la economía de la Unión Europea han desembocado en distintas transformaciones del sector minorista (Comisión Europea, 2013):

- Han aparecido cadenas de establecimientos de venta que comercializan múltiples líneas de productos.

- La integración vertical ha permitido a los minoristas desarrollar una distribución y una logística más eficientes.

- Los grandes grupos de distribución han obtenido economías de escala significativas en el proceso de aprovisionamiento, lo que aumenta su poder de negociación frente a los proveedores.

- Las compras centralizadas han llevado a una internacionalización progresiva de la oferta, tanto dentro de la Unión Europea como en terceros países (entre las diez principales empresas de distribución, cinco tienen su origen en Europa).

- Se han constituido asociaciones de compra y grupos de minoristas independientes con el propósito de poner en común sus conocimientos técnicos y conseguir economías de escala (especialmente en los procesos de aprovisionamiento).

- Por último, los minoristas han incrementado su control sobre la cadena de suministro para responder mejor a la demanda de los consumidores (por ejemplo, a través de las marcas propias o marcas del distribuidor).

La Comisión Europea viene reconociendo durante años el importante papel del sector del comercio y la distribución en el mercado único. En distintas comunicaciones, proporciona un análisis de la aportación económica, social y medioambiental del sector comercial y, además, considera las tendencias recientes y el impacto de los nuevos escenarios en los niveles de competencia.

En este contexto, la Comisión Europea (2013) establece que los sectores comerciales minorista y mayorista están llamados a desempeñar un papel esencial en el 
fomento del crecimiento y la creación de empleo dentro de la Estrategia Europa 2020: forman parte de los sectores fundamentales que pueden impulsar la transición a una economía y a unos modelos de consumo más sostenibles; la eficiencia en este sector tiene repercusiones sobre la competencia, la innovación, las tendencias de los precios y la competitividad.

Los objetivos de la Estrategia Europa 2020 (incremento de la tasa de empleo, aumento de la inversión en $\mathrm{I}+\mathrm{D}$, reducción de contaminantes, mayor protagonismo de las energías renovables, eficiencia energética, minoración de la tasa de abandono escolar, mayor participación de población con estudios superiores y reducción de habitantes en riesgo de pobreza y exclusión social) enlazan desde distintos frentes con el sector comercial. Esto es, el logro de un comercio y de una distribución inteligentes, sostenibles e integradores puede contribuir directamente al éxito de la estrategia para la Europa de 2020.

\subsection{Comercio e innovación}

Dentro de la Estrategia Europea 2020, se establece como objetivo aumentar el nivel combinado de inversión pública y privada en I+D al 3 por 100 del PIB. Con carácter general, la innovación se ha convertido en un factor esencial para lograr el éxito de las empresas y, consecuentemente, en un factor clave en el crecimiento de cualquier economía. En las actividades de distribución comercial, la innovación es un concepto que se utiliza como respuesta a las demandas de los consumidores, a las exigencias del mercado y a la competencia global (el entorno competitivo obliga a las empresas a innovar más rápida y eficientemente) (Casares y Martín, 2011).

La Comisión Europea ha calificado a las empresas comerciales de multiplicadores de innovación, puesto que las empresas comerciales están en contacto directo con los consumidores, perciben mejor las nuevas exigencias y, por tanto, consiguen adecuar la oferta a una demanda variable y multiforme. El sector comercial necesita aumentar su nivel de inversión en tecnologías y prácticas innovadoras para seguir mejorando la competitividad en toda la cadena de suministro, incluidas la logística y el transporte, la eficiencia energética, el envasado, la eliminación de residuos y el reciclado de productos.

En los últimos años, la participación del sector comercial en el proceso de innovación ha generado distintas posturas e interpretaciones, sobre todo, por la creciente cuota que representan los productos con marca del distribuidor frente a los productos de los fabricantes. En este contexto, se plantea cual debe ser el origen de la innovación (fabricantes o distribuidores). Como resultado final, las innovaciones en gran consumo se asocian especialmente a marca del fabricante mientras que las marcas de distribuidor tiene una participación más reducida.

Así pues, a pesar de que la Comisión Europea reconoce que las relaciones entre empresas comerciales y sus proveedores, basadas en una relación de contratación, 
constituyen relaciones estables y duraderas, también apunta a la necesidad de atender al impacto que los productos con marcas del distribuidor están teniendo sobre los proveedores, la competencia, la innovación y la libertad de elección de los consumidores.

Desde un prisma más amplio, el sector comercial también desarrolla innovación en sus procesos (por ejemplo, de marketing o de gestión) que suponen un factor clave de desarrollo y modernización del sistema distributivo. En este sentido, se distinguen los siguientes tipos de innovación:

- Innovación de proceso: Supone la generación de un método de distribución nuevo o con un alto grado de mejora al incluir nuevas técnicas, equipo o tecnologías.

- Innovación en marketing: Se asocia con la implementación de un nuevo método de comercialización (mejoras de producto, presentaciones, emplazamiento y posicionamiento, promoción o precio).

- Innovación organizacional: Utilización de un nuevo método de organización aplicado a las prácticas de negocio, al lugar de trabajo o a las relaciones externas de la empresa.

Finalmente, otro aspecto importante que también hay que tener en cuenta, es la necesidad de otorgar una protección suficiente a la innovación en algunos ámbitos de la producción para protegerla frente a determinadas prácticas desleales (imitaciones o copias) que repercuten negativamente en el balance final de la innovación en el sector comercial.

\subsection{Comercio, energía y residuos}

La Estrategia Europea 2020 plantea un triple objetivo en referencia a las externalidades negativas sobre el medio ambiente: reducir las emisiones de gases de efecto invernadero al menos en un 20 por 100 en comparación con los niveles de 1990; aumentar hasta el 20 por 100 la cuota de energías renovables en el consumo energético final, y además avanzar hacia un incremento del 20 por 100 de la eficiencia energética.

En esta dirección, diferentes estudios han manifestado recientemente que las políticas públicas que promueven estilos de vida más sostenibles, entre los que se encontraría la comercialización de productos de gran consumo, aproximan hacia un medioambiente más sostenible e incrementan la calidad de vida (se ha denominado Happiness Economics, por ejemplo, en distintos enfoques realizados en la London School of Economics).

En el sector del comercio existe una larga tradición de responsabilidad social de las empresas. De hecho, durante muchos años las empresas dedicadas al comercio 
han puesto en marcha y han llevado a cabo numerosas prácticas responsables que no solo se refieren a cuestiones sociales y medioambientales, sino también a otros ámbitos como salud, seguridad de los productos y asuntos relacionados con la cadena de suministro o con el compromiso local.

Resulta obvio que el comercio y la distribución tienen un impacto ambiental significativo en el desenvolvimiento cotidiano de sus actividades:

- Necesidad de desplazamientos geográficos por parte de los consumidores.

- Consumo de energía para atender a las necesidades de los establecimientos (alumbrado, refrigeración, calefacción, climatización, etcétera).

- Generación (y tratamiento) de cantidades muy importantes de excedentes y residuos (folletos publicitarios, envases, residuos alimentarios, materiales eléctricos y electrónicos, etcétera).

- Dependencia del tráfico de mercancías considerando, además, que gran parte de los suministros se efectúan por carretera (escasa diversificación fluvial y ferroviaria).

- Congestión, contaminación, ruidos y otros efectos negativos sobre la ciudad derivados del transporte y suministro de mercancías en los establecimientos de ámbito urbano.

- Generación de externalidades negativas que se derivan de las actividades logísticas que desarrollan las grandes cadenas de distribución.

- El sistema distributivo facilita la elección de los productos pero se centra fundamentalmente en el proceso de compra, favoreciendo la satisfacción inicial y descuidando la vida avanzada del producto (nivel de reciclaje del envase, duración del rendimiento, accesibilidad al servicio técnico y a recambios, valor en el momento de su posible reventa como producto usado, servicios posventa).

Conforme a todo lo anterior, la Comisión Europea ha marcado como objetivo sobre el sector comercial hacer que las cadenas de suministro sean más respetuosas y sostenibles con el medio ambiente mediante el empleo de todos los medios que permitan reducir el uso de energía y la generación de sustancias contaminantes.

Así pues, un sector del comercio y de la distribución más justo y eficaz para las generaciones futuras debe atender plenamente al impacto ambiental:

- Ofrecer una amplia selección de productos respetuosos con el medio ambiente.

- Considerar a los aprovisionamientos que procedan del comercio justo.

- Permitir que las empresas éticas, incluidas las de terceros países, accedan al mercado interior.

- Tomar como base un consumo reducido de energía (por ejemplo, en logística o recogida y reciclaje de residuos). 
Como resultado de lo anterior, la nueva concepción de la competencia no solo comprende factores económicos, sino que también atiende a criterios sociales, éticos y ambientales. Se reconoce, por tanto, la importancia del comercio y la distribución a la hora de fomentar unas pautas más sostenibles de consumo y producción en la Unión Europea.

En esta interpretación emergente, resulta necesario garantizar la coherencia política entre los objetivos medioambientales y otros objetivos políticos (por ejemplo, consolidar el mercado interior). A lo largo de los últimos años han surgido conflictos y se ha hecho cada vez más frecuente invalidar los principios del mercado interior alegando la protección medioambiental. Por otra parte, también se advierte de un posible traspaso de la responsabilidad del productor al comerciante.

\subsection{Comercio y formación}

En cuanto a la vertiente formativa, la Estrategia Europea 2020 marca, por un lado, reducir la tasa de abandono escolar a menos del 10 por 100 y, por otro, aumentar al menos hasta el 40 por 100 el porcentaje de la población entre 30 y 34 años de edad que haya terminado estudios superiores.

La constante innovación, y el consiguiente aumento de los niveles de automatización que ha experimentado el sector minorista, han exigido que los trabajadores actualicen sus cualificaciones para adaptarse a la evolución de sus tareas.

Sin embargo, la situación muestra como casi un tercio de los empleados del comercio minorista carecen de cualificaciones o está semicualificado. Si a menudo la capacidad de inserción de los trabajadores con un bajo nivel de cualificaciones se ha marcado como una característica positiva del sector del comercio, existe también una contrapartida negativa que se plasma en una mayor dificultad del sector para adoptar con rapidez nuevas soluciones tecnológicas. Esto es, la falta de competencias en la utilización de las tecnologías de la información y de la comunicación reduce la productividad del sector (por ejemplo, la importancia relativa de los pequeños establecimientos dificulta una inversión suficiente en formación continua).

Por otra parte, la evolución reciente de las actividades distributivas ha dado lugar a una clara divergencia entre las necesidades de cualificación de las empresas y las competencias consolidadas por los trabajadores del sector. El problema reside en el desfase entre las cualificaciones adquiridas y las necesidades de las empresas y, por tanto, adquieren sentido todos los esfuerzos que se vienen haciendo durante años en este ámbito como, por ejemplo, la COM (2012) 669 final Un nuevo concepto de educación, que tiene como objetivo poder contar con una mano de obra adecuada y convenientemente cualificada, multiplicando los esfuerzos para modernizar los sistemas de educación y formación. En este sentido, parece fundamental adecuar las cualificaciones profesionales requeridas y las competencias de los empleados y, además, el compromiso de las empresas no puede limitarse a determinar los contenidos de la formación, sino que deben también efectuar inversiones específicas. 
Tomando como referencia las actuaciones del Consejo Sectorial Europeo de Capacitación en el Sector del Comercio, los Estados miembros han de constituir organismos bilaterales entre los interlocutores sociales para el desarrollo de la formación profesional (adecuación de las cualificaciones a las necesidades de las empresas, planes de formación, financiación, determinación de las necesidades de formación, puesta en práctica e intercambio de información sobre la formación).

Por tanto, a modo de conclusión, entre los principales retos del sector, se encuentran la mejora de las condiciones de trabajo, la lucha contra la economía sumergida y el mantenimiento de los niveles de empleo y de competitividad mediante una mejor adecuación entre las necesidades de los minoristas y la cualificación de sus empleados; destaca, a tal fin, la necesidad de invertir en la formación y en el desarrollo de competencias, lo que contribuirá a que el sector se adapte con rapidez a las nuevas tecnologías.

\subsection{Comercio y exclusión social}

La Estrategia Europea 2020 también marca como fin conseguir apartar al menos a 20 millones de personas del riesgo de pobreza y exclusión social.

La crisis económica que han sufrido las economías europeas durante los últimos años ha acentuado la asimetría en la distribución de renta y, por tanto, ha supuesto un incremento de la exclusión social o, en otras palabras, un aumento de la población en riesgo de pobreza.

Un mercado interior del comercio y de la distribución más justo y eficaz debe ser capaz de contribuir a mejorar la productividad y, al mismo tiempo, las condiciones laborales. El sector del comercio es una de la principales fuentes de empleo de la economía europea, el canal de acceso al mercado laboral para muchos jóvenes (un 30 por 100 de los asalariados del sector cuenta con menos de 30 años) que carecen de cualificaciones o están poco cualificados. Además, se caracteriza por ser un recurso relevante el trabajo para el tiempo parcial (30 por 100 de la mano de obra). Y, finalmente, cabe señalar también que es el sector que registra la mayor proporción de empleo femenino (la media europea está cercana al 50 por 100 aunque en algunos países es superior al 60 por 100).

En el sector comercial y de la distribución se advierten distintos desequilibrios que pueden mermar, en mayor o menor medida, la equidad del mercado interior:

- La existencia de economía sumergida que, aunque es difícil de medir, tiene una repercusión negativa sobre las condiciones de trabajo, sobre todo en la pequeñas empresas comerciales, en las cuales es más difícil controlar de forma efectiva el cumplimiento de la legislación y los convenios colectivos. En este caso, resulta complejo erradicar las prácticas negativas que repercuten en las condiciones laborales (por ejemplo, en la protección de la salud o de la seguridad en el trabajo). 
- Los trabajadores de la economía informal quedan excluidos de las coberturas sanitarias y sociales pero, además, no tienen la posibilidad de acceder a formación (evidentes consecuencias negativas para sus oportunidades de adquirir competencias y cualificaciones profesionales).

- El trabajo irregular también genera una situación de competencia desleal entre empresas en lo que respecta a los costes del trabajo. El trabajo no declarado y la economía sumergida generan, por otro lado, competencia desleal entre las empresas a causa de la evasión fiscal y contributiva.

- La exclusión en las actividades comerciales también llega por la vía de los salarios (generalmente, más reducidos que en otras actividades del sector servicios) y por las condiciones laborales de ciertos colectivos (especialmente, jóvenes y mujeres).

- El consumidor no dispone de información sobre las prácticas o conducta del comerciante en términos de responsabilidad social y, por tanto, no cuenta con suficiente criterio para elegir productos, enseñas o formatos comerciales apelando al compromiso de integración social.

- La gran competencia sobre los precios es fuente de presiones para flexibilizar el horario laboral de los empleados llegando, en algunas ocasiones, a ser incompatible con aspectos personales (por ejemplo, la conciliación familiar).

La crisis económica y la contención del consumo se han saldado con procesos de cierres, reorganizaciones, fusiones y adquisiciones de las actividades comerciales. Por lo tanto, ha aumentado el riesgo de contar con personas que permanezcan excluidas del mercado laboral (desempleados de larga duración, trabajadores de edad avanzada, inmigrantes y personas con discapacidad). Para luchar contra esta exclusión social, debe fomentarse el empleo autónomo y el desarrollo de las competencias profesionales de los empleados permitiendo la reinserción de las personas en el mercado de trabajo.

En relación a este objetivo de la Estrategia Europea 2020, pueden plantearse dos temas colaterales que en los últimos años están adquiriendo protagonismo:

- El primero es la importancia de las marcas del distribuidor que se defienden, entre otros aspectos, porque permite a los ciudadanos con ingresos más bajos acceder a una gama más amplia de productos y a unos precios más ajustados.

- En segundo lugar, en época de crisis crece la preocupación por el desperdicio alimentario. FAO y Parlamento Europeo han solicitado a los actores de la cadena alimentaria colaboración activa para solucionar el problema de las pérdidas y desperdicios de alimentos. Este problema afecta al desarrollo sostenible y se estima que en 2020, se puede llegar a 126 millones de toneladas de residuos de alimentos. Por ejemplo, en Francia cada habitante tira por término medio 20 kilos de alimentos al año, al mismo tiempo 16 millones de personas dependen de la ayuda alimentaria. Otro ejemplo: en 2015, las empresas de Mercabarna 
habían donado al Banco de Alimentos 876.000 toneladas, un 146 por 100 más que en 2014.

\subsection{Comercio y empleo}

En la Estrategia Europea 2020, se marca como objetivo aumentar la tasa de empleo de la población entre 20 y 64 años de edad al menos hasta el 75 por 100. Posiblemente, este sea el objetivo más importante y, al mismo tiempo, aparece interrelacionado con los que se han analizado anteriormente. Innovación, eficiencia energética, formación e inclusión social están directamente relacionados con el mercado de trabajo y la posibilidad de que todos los individuos que lo busquen encuentren un empleo en unas condiciones oportunas.

Tradicionalmente, se ha estudiado al sector distributivo como «refugio» de trabajadores de otros sectores que se instalan en el comercio. Por ejemplo, el sector distributivo «recoge» grupos de jóvenes o mujeres desanimadas, y en general poco cualificadas, que salen del mercado de trabajo en épocas de crisis y que se incorporan cuando mejora la situación laboral, «refugiándose» en aquellas actividades económicas que mejor se adaptan a sus características (empleo poco cualificado, relativamente bien remunerado, temporal e incluso parcial).

Otro elemento determinante del tipo de empleo de este sector es la dimensión de las empresas. Las actividades comerciales representan un porcentaje elevado del tejido empresarial europeo aunque se trata de una de las ramas con mayor nivel de atomización. Esta circunstancia refleja la gran importancia que en este sector tiene el empleo no asalariado y la gran concentración que el asalariado presenta.

La importancia del empleo a tiempo parcial es razonable al considerar las características del mercado de trabajo del comercio: desde el lado de la oferta, vuelve a destacarse la alta participación de mujeres y jóvenes y, desde el lado de la demanda, las propias características de la distribución como la necesidad de mano de obra flexible que complemente el empleo a tiempo completo para hacer frente por un lado, a los amplios horarios de apertura y, por otro, a las fluctuaciones en el nivel de ventas de los establecimientos.

A modo de resumen de este apartado, y como punto de referencia para todos los objetivos de la Estrategia Europea 2020, el Gráfico 1 refleja un resumen estadístico del empleo en la distribución comercial para el ámbito de la Unión Europea. 


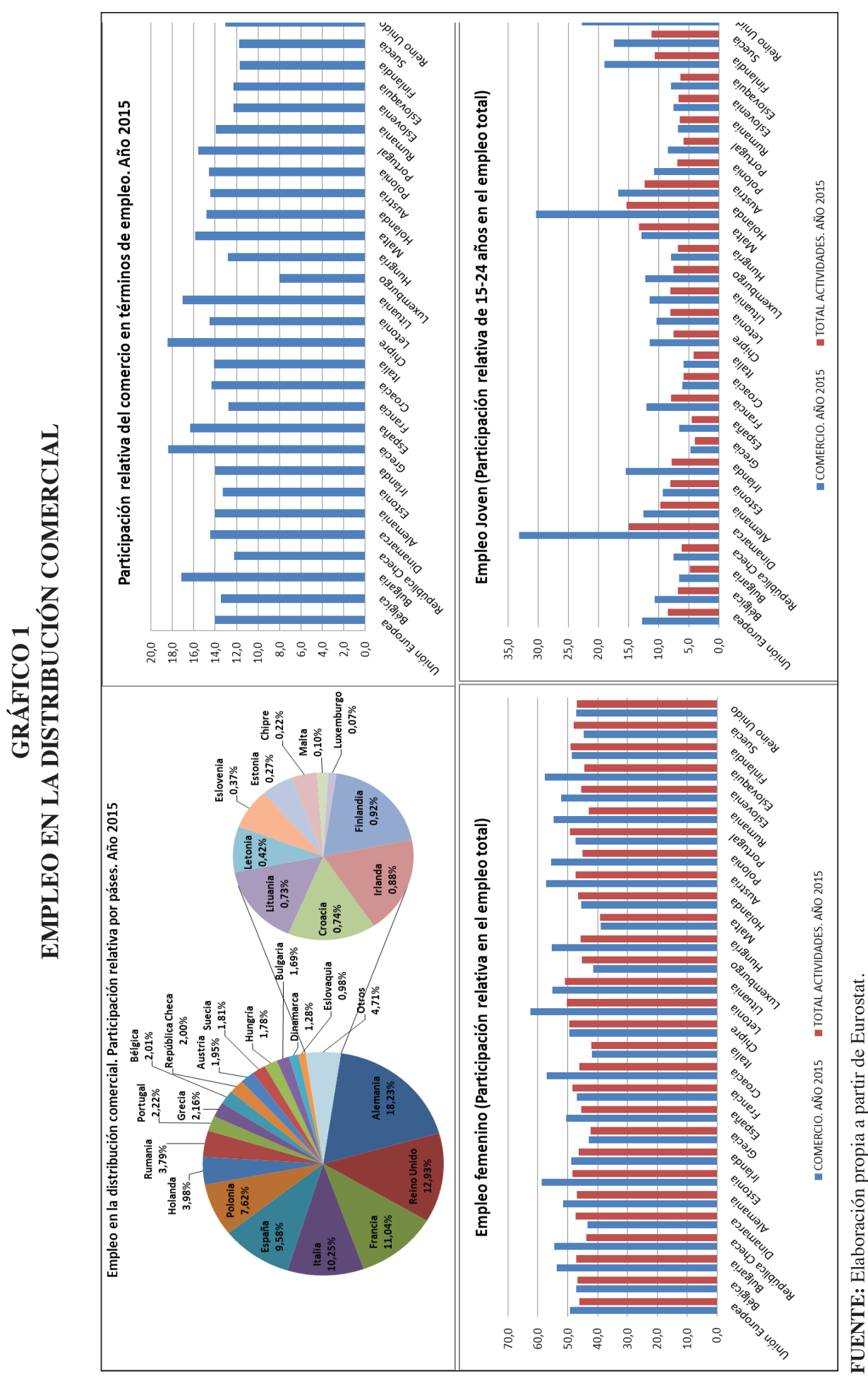




\section{Actuaciones públicas y distribución comercial en la Europa 2020}

Un planteamiento teórico de cualquier actuación pública en distribución comercial hace referencia a una política sectorial constituida por fragmentos de políticas instrumentales -financiera, fiscal, urbanística, etcétera-, con claras intenciones finalistas en lo relativo, sobre todo, a objetivos de política económica alguno de ellos analizados en el epígrafe anterior del artículo -empleo, innovación, formación, medio ambiente, etcétera-. Para la consecución de estos objetivos, las actuaciones públicas se desenvuelven en torno a cinco grandes grupos de instrumentos o áreas de actuación como son la regulación comercial, el abastecimiento de productos básicos, la estabilidad de precios, la protección del consumidor y la modernización del sector.

Por tanto, en el análisis de la política de comercio interior en la Unión Europea habría que distinguir entre políticas de carácter general -política de protección a los consumidores, política de competencia, política de transporte, política medioambiental, etcétera- que afectan al comercio y políticas específicas de actuación en el sector distributivo -Libro Verde de Comercio de 1996, Libro Blanco del Comercio de 1999, Plan de Acción Europeo para el Comercio Minorista de 2013, etcétera-, sin olvidar las actuaciones llevadas a cabo en el sector en cada uno de los países miembros.

En cuanto a instrumentos, en la Unión Europea la regulación de las actividades comerciales es competencia de los Estados miembros aunque ha de cumplir la legislación comunitaria, sobre todo en lo referido a competencia, libertad de establecimiento y libre circulación de mercancías y servicios -en el marco de la consecución de un mercado interior de servicios minoristas en la Unión Europea-. Además, en el ámbito de la política regional y estructural, tanto los Fondos Estructurales como las distintas Iniciativas Comunitarias han contribuido notablemente a la modernización del sector.

En esta política de comercio en la Unión Europea destacan, en el ámbito regulatorio, la Directiva de Servicios o las iniciativas en el ámbito de pagos. La Directiva de Servicios (Directiva 2006/123/CE de 12 de diciembre) supone hacer efectivas dos libertades fundamentales de la Unión Europea como son la libertad de establecimiento de los prestadores de servicios y la libre circulación de servicios entre los Estados miembros; es decir, con esta nueva regulación se pretende facilitar el establecimientos de las empresas europeas en otros Estados miembros y fomentar el normal desarrollo de su actividad en un país diferente a aquél en que están establecidos. No obstante, en algunos países, la nueva normativa faculta para establecer regímenes de autorización específicos cuando concurran razones imperiosas de interés general, como la protección del medio ambiente y del entorno urbano, la ordenación del territorio y la conservación del patrimonio histórico y artístico, y siempre que estas autorizaciones se concedan sobre la base de criterios proporcionados, no discriminatorios, claros e inequívocos, objetivos, hechos públicos con antelación, predecibles, transparentes y accesibles. 
En el marco de la Estrategia Europa 2020, como ya se ha hecho referencia, la Unión Europea atiende al importante desempeño económico, ambiental y social del sector minorista y, por tanto, a través de sus actuaciones públicas pretende mejorar sus condiciones de competitividad. Con este objetivo, la Comisión Europea aprobó el 31 de enero de 2013 una estrategia global -Plan de Acción Europeo para el Comercio Minorista- centrada en cinco ejes o prioridades fundamentales y once acciones que tienen un impacto directo en el comercio minorista y abordan los distintos problemas identificados (véase Cuadro 1). El conjunto de acciones que engloban este Plan deberán ser impulsadas por la Comisión y los Estados miembros, debiendo presentar un informe conjunto del Progreso del Plan. Asimismo, la Comisión creó en diciembre de 2013 un Grupo sobre Competitividad en el Comercio Minorista de carácter permanente en el que participan los Estados miembros y todas las partes interesadas de la cadena de suministro (minoristas grandes y pequeños, proveedores, cooperativas, grupos de interés vinculados con el consumo, etc.) con la finalidad de avanzar en la problemática de las barreras al mercado único al por menor y del potencial desarrollo del sector. Por tanto, una aproximación a la política de comercio minorista en el marco de la Europa 2020 se podría concretar en el siguiente elenco de temas clave o actuaciones públicas relevantes:

- Barreras a un mercado interior de la distribución comercial. Las actuaciones por parte de los decisores públicos comunitarios deberán fomentar la competencia y garantizar que las empresas comerciales puedan realizar sus operaciones transfronterizas de una manera similar a las desarrolladas en su país de origen; adquieren especial protagonismo la regulación comercial vinculada con la apertura de establecimientos comerciales, las restricciones operativas (ventas a pérdida, ventas especiales, actividades de promoción de ventas, horarios comerciales, etcétera) y el etiquetado de productos.

- Comercio electrónico. El desarrollo del comercio electrónico en la Unión Europea se está viendo limitado por: la fragmentación jurídica en múltiples áreas; los problemas asociados a falta de confianza (pagos, entregas, devoluciones, protección de datos, etcétera) por parte de empresas -fundamentalmente pequeñas y medianas empresas- y consumidores; y el desarrollo de ciertas inversiones (plataformas, banda ancha, etcétera). Por tanto, los decisores públicos tienen que trabajar para minimizar estos obstáculos, permitiendo desarrollar el potencial de este tipo de comercio. Asimismo, deberán tener en cuenta las posibles prácticas desleales y la problemática de la fiscalidad.

- Apoyo a pequeñas y medianas empresas comerciales. Los decisores públicos comunitarios deben prestar especial atención a las acciones destinadas a apoyar al pequeño comercio, permitiendo que siga desempeñando la función social y económica ya analizadas, y ayudándole a hacer frente a los retos originados por los cambios en el sector: operativos (economías de escala, poder de negociación en la cadena de suministro, innovación, etcétera), financieros (acceso reducido y con dificultades), capital humano (cualificación, formación digital, 


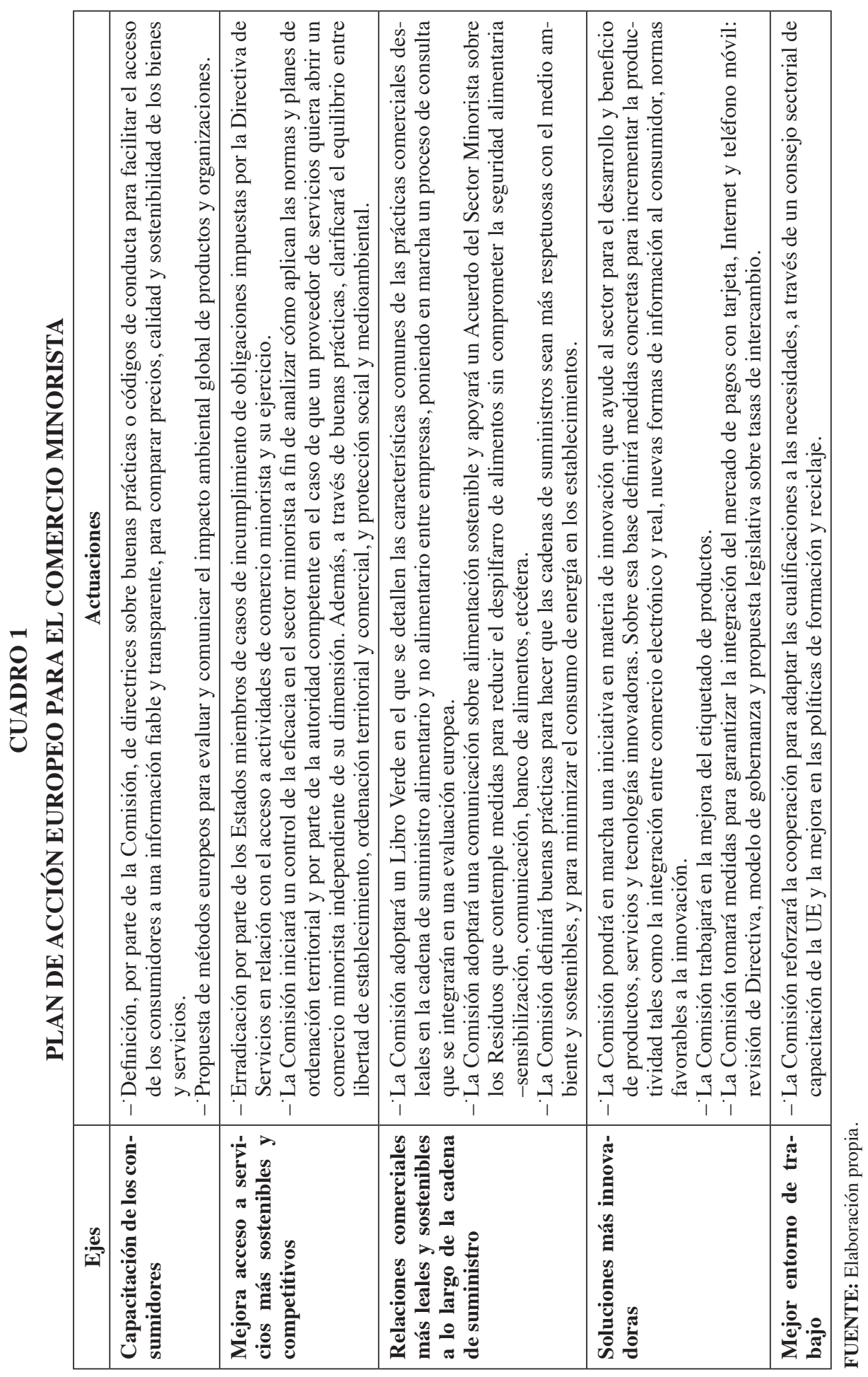


etcétera), crisis económica y nuevos competidores (abandono del centro urbano «muerte de la calle», grandes empresas globalizadas con menores retornos del beneficio al ámbito local, etcétera), nuevo entorno regulador, costes burocráticos, etcétera.

- Innovación. Los decisores públicos deben ser conscientes del carácter innovador del sector, como anteriormente se ha contemplado, debiendo establecer un conjunto de actuaciones destinadas a reconocer y fomentar dicho papel, entre las que se podrían destacar: apoyo financiero -incorporación del sector a programas europeos ya existentes, reconociendo las especificidades del mismo-, participación de las empresas minoristas como asesores en los programas de innovación europeos, o establecimiento de una estructura permanente que permita el intercambio de ideas y experiencias entre los distintos agentes del sector. Para facilitar estas actuaciones, se ha creado por parte de la Comisión Europea un Grupo de Expertos de Alto Nivel sobre la Innovación en el Sector Minorista.

- Entorno de trabajo. Los decisores públicos deben abordar un política de cualificación en el comercio minorista que permita adaptar los recursos humanos a los nuevos requerimientos (internacionalización, digitalización. etcétera) tanto en la formación inicial como en la formación continua. Además, en el marco del diálogo social, las actuaciones públicas deben estar destinadas a fomentar tres grandes objetivos de la Europa 2020: empleo, exclusión social y abandono escolar.

- Relaciones comerciales a lo largo de la cadena de suministro. La concentración del sector puede tener repercusiones sobre el poder de negociación de los distintos eslabones del canal comercial, debiendo los decisores públicos garantizar la existencia de una competencia efectiva en el sector comercial (véase Cuadro 2).

Para finalizar, en el marco del Semestre Europeo, la Comisión Europea ha recomendado a los países miembros continuas mejoras en el entorno regulador del sector minorista para que contribuya a la consecución de los objetivos de la Europa 2020 (fundamentalmente el aumento de la flexibilidad y la competitividad de los mercados de bienes y de servicios). En el Cuadro 3 se han recogido las principales recomendaciones realizadas en el últimos años pudiéndose observar que, según la Comisión Europea, se han producido reformas en el marco regulador del sector minorista requiriendo, no obstante, mayores esfuerzos. 


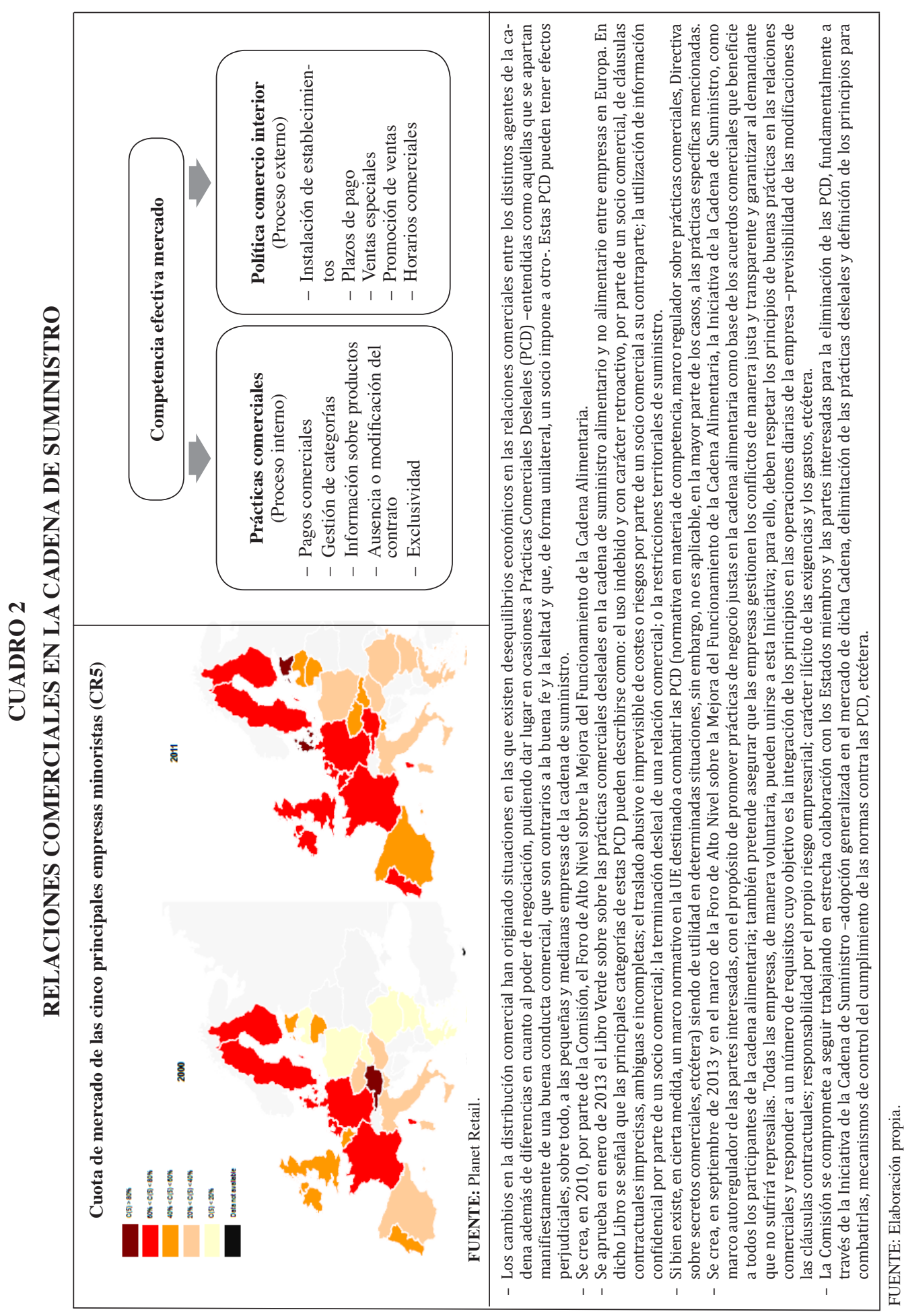




\section{CUADRO 3}

\section{ACTUACIONES PÚBLICAS EN EL COMERCIO MINORISTA. SEMESTRE EUROPEO}

\begin{tabular}{|c|c|}
\hline \multicolumn{2}{|r|}{ Recomendaciones de la Comisión Europea a los Estados miembros } \\
\hline $\begin{array}{l}\text { Semestre } \\
\text { Europeo } \\
2012\end{array}$ & $\begin{array}{l}\text { - Se recomienda, para algunos Estados miembros, una mayor independencia de los reguladores. } \\
\text { - Se hace referencia a la aplicación de la Directiva de Servicios, recomendando la supresión de las } \\
\text { restricciones injustificadas o desproporcionadas para la prestación de servicios además de una apli- } \\
\text { cación más ambiciosa de la misma. } \\
\text { - Se recomienda establecer medidas para mejorar la competencia y la competitividad en el sector } \\
\text { minorista entre las que destacan la eliminación de obstáculos a la entrada y a la salida de empresas. }\end{array}$ \\
\hline $\begin{array}{l}\text { Semestre } \\
\text { Europeo } \\
2013\end{array}$ & $\begin{array}{l}\text { - Se hace referencia al gran potencial económico y de creación de empleo del sector de los servicios } \\
\text { que todavía está sin explotar. } \\
\text { - Se recomiendan medidas concretas para mejorar sustancialmente la competencia en el sector ser- } \\
\text { vicios, eliminando, por ejemplo, los obstáculos al comercio minorista. La plena aplicación de la } \\
\text { Directiva de Servicios puede desempeñar un papel importante en el desarrollo de los servicios trans- } \\
\text { fronterizos y contribuir a aumentar la productividad en los mercados nacionales. } \\
\text { - Además, se insta la urgencia de estos mayores esfuerzos para crear las condiciones que favorezcan } \\
\text { el desarrollo empresarial, la situación de los consumidores y la creación de empleo; por ejemplo, aún } \\
\text { son necesarias mejoras sustanciales en servicios clave como el comercio minorista. }\end{array}$ \\
\hline & $\begin{array}{l}\text { - Se constata que unos servicios que funcionen correctamente son esenciales para el crecimiento eco- } \\
\text { nómico, tanto directamente, como fuente fundamental de puestos de trabajo, como indirectamente, } \\
\text { pues representan un insumo cada vez más importante para otros sectores y para la productividad de } \\
\text { la economía. } \\
\text { - Se pone de manifiesto que contar con un marco de competencia eficiente resulta fundamental para } \\
\text { el funcionamiento de los mercados de bienes y servicios. A pesar de que se han tomado algunas } \\
\text { medidas encaminadas a la aplicación de nuevas leyes en materia de competencia y al refuerzo de los } \\
\text { poderes de las autoridades reguladoras y de defensa de la competencia nacionales, se recomienda, } \\
\text { en algunos países, reforzar la independencia de las autoridades de competencia, especialmente do- } \\
\text { tándolas de recursos humanos y financieros suficientes. }\end{array}$ \\
\hline & $\begin{array}{l}\text { - Se refleja la necesidad en muchos Estados miembros de una aplicación ambiciosa de reformas es- } \\
\text { tructurales para aumentar la flexibilidad y la competitividad de los mercados de productos y de } \\
\text { servicios. El Estudio Prospectivo Anual sobre el Crecimiento } 2015 \text { destacó en particular las seis } \\
\text { barreras desde una perspectiva europea: i) unos requisitos de autorización desproporcionados e in- } \\
\text { justificados, entre otros, los referentes a la forma jurídica y a la participación accionarial; ii) la falta } \\
\text { de claridad de la legislación nacional por lo que atañe a las normas aplicables a las empresas que } \\
\text { prestan servicios transfronterizos; iii) la inexistencia de reconocimiento mutuo; iv) la falta de agi- } \\
\text { lidad en los procedimientos administrativos; v) los progresos desiguales en el proceso en curso de } \\
\text { evaluación mutua de las reglamentaciones profesionales y las reformas de las profesiones reguladas; } \\
\text { vi) la persistencia de obstáculos a la libre circulación de bienes. } \\
\text { - Se señala la existencia de Estados miembros donde hay margen para aumentar la competencia del } \\
\text { sector servicios, en concreto señalan que en Francia existe dicho margen para el comercio minorista } \\
\text { ya que la aplicación de una serie de normativas en el mismo limita la actividad económica. Se con- } \\
\text { templa, asimismo, que en Dinamarca, el crecimiento de la productividad se ha visto afectado por } \\
\text { los obstáculos a la entrada y la carga normativa en los sectores de servicios orientados al mercado } \\
\text { nacional, entre los que aparece el comercio minorista. }\end{array}$ \\
\hline & $\begin{array}{l}\text { - Se señalan los progresos realizados por los Estados miembros para la mejora del marco regulador } \\
\text { del sector minorista; sin embargo, se constata el requerimiento de un mayor esfuerzo, fundamental- } \\
\text { mente en la mejora de las condiciones de establecimiento. } \\
\text { - Se destacan los avances realizados en países como Finlandia que ha derogado la Ley que regulaba } \\
\text { los horarios de apertura y que, mediante modificaciones de las normas de ordenación del territorio, } \\
\text { ha avanzado en la mejora de las condiciones de establecimiento del sector minorista; y también, } \\
\text { España, con la reforma del sector minorista de } 2014 \text { que sentó las bases para unas mejores } \\
\text { condiciones de establecimiento y de explotación -no obstante, se observa que los beneficios de esa } \\
\text { reforma solo se percibirán en la práctica cuando las Comunidades Autónomas adopten los actos de } \\
\text { ejecución necesarios-. Asimismo, se señala que Italia ha acometido reformas dirigidas a reducir las } \\
\text { restricciones comerciales y a ampliar la competencia: se trata del paquete de liberalización de 2012, } \\
\text { del plan de privatización y de la legislación de competencia de fecha más reciente. }\end{array}$ \\
\hline
\end{tabular}

FUENTE: Elaboración propia. 


\section{Conclusiones}

Las funciones y servicios que prestan las empresas comerciales en la intermediación entre la producción y los consumidores están basadas en la necesidad de salvar las separaciones existentes entre la oferta económica y la demanda final. En este contexto, el comercio minorista se configura como un sector esencial puesto que tiene implicaciones para la innovación, la evolución de los precios o la competitividad empresarial.

La Comisión Europea reconoce esta significatividad y, por tanto, establece que los sectores comerciales minorista y mayorista están llamados a desempeñar un papel esencial en el fomento del crecimiento y la creación de empleo dentro de la Estrategia Europa 2020. Este trabajo, en un primer apartado, ha planteado la relación entre los objetivos de la Estrategia Europa 2020 y distintas áreas del sector comercial. El logro de un comercio y de una distribución inteligentes, sostenibles e integradores puede contribuir directamente al éxito de la estrategia para la Europa de 2020:

- Comercio e innovación. La Comisión Europea ha calificado a las empresas comerciales de multiplicadores de innovación, puesto que las empresas comerciales están en contacto directo con los consumidores, perciben mejor las nuevas exigencias y, por tanto, consiguen adecuar la oferta a una demanda variable y multiforme.

- Comercio, energía y residuos. Un sector del comercio y de la distribución más justo y eficaz para las generaciones futuras debe atender plenamente al impacto ambiental y, por tanto, ofrecer una amplia selección de productos respetuosos con el medio ambiente; considerar a los aprovisionamientos que procedan del comercio justo; permitir que las empresas éticas, incluidas las de terceros países, accedan al mercado interior; $y$, tomar como base un consumo reducido de energía (por ejemplo, en logística o recogida y reciclaje de residuos).

- Comercio y formación. El problema del desfase entre las cualificaciones adquiridas por los trabajadores y las necesidades de las empresas comerciales debe resolverse multiplicando los esfuerzos para modernizar los sistemas de educación y formación. En este sentido, parece fundamental adecuar las cualificaciones profesionales requeridas y las competencias de los empleados y, además, el compromiso de las empresas no puede limitarse a determinar los contenidos de la formación, sino que deben también efectuar inversiones específicas.

- Comercio y exclusión social. La crisis económica y la contención del consumo se ha saldado con procesos de cierres, reorganizaciones, fusiones y adquisiciones de las actividades comerciales. Por lo tanto, ha aumentado el riesgo de contar con personas que permanezcan excluidas del mercado laboral (desempleados de larga duración, trabajadores de edad avanzada, inmigrantes y personas con discapacidad). Para luchar contra esta exclusión social, debe fomentarse el empleo autónomo y el desarrollo de las competencias profesionales de los empleados permitiendo su reinserción laboral. 
- Comercio y empleo. Aumentar la tasa de actividad en el mercado de trabajo europeo es, sin ninguna duda, el objetivo más importante y, al mismo tiempo, recoge todos los aspectos que se han tratado en el resto de fines (innovación, eficiencia energética, formación e inclusión social).

La segunda parte de este trabajo ha profundizado en las actuaciones públicas que se llevan a cabo desde la Unión Europea para la consecución de los objetivos citados. Para lograr estos objetivos, las actuaciones públicas se desenvuelven en torno a cinco grandes grupos de instrumentos o áreas de actuación como son la regulación comercial, el abastecimiento de productos básicos, la estabilidad de precios, la protección del consumidor y la modernización del comercio interior.

En el marco de la Estrategia Europa 2020, la Comisión Europea aprobó en 2013 el Plan de Acción Europeo para el Comercio Minorista centrado en cinco ejes o prioridades fundamentales y once acciones que tienen un impacto directo en el comercio minorista y abordan los distintos problemas identificados. Asimismo, la Comisión también creó en 2013 un Grupo sobre Competitividad en el Comercio Minorista con la finalidad de avanzar en la problemática de las barreras al mercado único al por menor y del potencial desarrollo del sector.

Desde una vertiente práctica, una aproximación a la política de comercio minorista en el marco de la Europa 2020 permite hacer referencia a seis elementos clave:

- Barreras a un mercado interior de la distribución comercial. Fomentar la competencia vinculada con la apertura de establecimientos comerciales, las restricciones operativas y el etiquetado de productos.

- Comercio electrónico. Minimizar los obstáculos que impiden el pleno desarrollo de esta modalidad de venta atendiendo, al mismo tiempo, a las posibles prácticas desleales y la problemática de la tributación.

- Apoyo a pequeñas y medianas empresas comerciales. Prestar asistencia a los pequeños empresarios comerciales en los retos operativos, financieros, formativos, normativos, burocráticos, vinculados con la crisis y nuevos competidores, etcétera.

- Innovación. Facilitar apoyo financiero, asegurar la participación de las empresas minoristas e impulsar el intercambio de ideas y experiencias entre los distintos agentes del sector.

- Entorno de trabajo. Abordar un política de cualificación tanto en la formación inicial como en la formación continua y, además, atender al empleo, la exclusión social y el abandono escolar.

- Relaciones comerciales a lo largo de la cadena de suministro. Garantizar la existencia de una competencia efectiva en todos los niveles del sector comercial. 
Para finalizar, en el marco del Semestre Europeo, la Comisión Europea también ha recomendado a los países miembros continuas mejoras en el entorno regulador del sector minorista para que el sector contribuya a las consecución de los objetivos de la Europa 2020.

\section{Referencias bibliográficas}

[1] ARANDA, E.; MARTÍN, V. J.; CASARES, J. y SANTOS, J. (2016). «Competencia efectiva en el sector de la distribución comercial. Un análisis comparado de la evolución internacional», en E. Aranda (dir.), J. R. Cuadrado y J. Casares (coords.), Política económica. Un contexto de crisis, recuperación e incertidumbres. Madrid: Civitas-Thomson Reuters.

[2] CASARES, J. y MARTÍN, V. J. (2011). «Creatividad, innovación y tecnología en la distribución comercial». Distribución y Consumo, 115, 5-25.

[3] CASARES, J. y MARTÍN, V. J. (2012). «Las metamorfosis en relación con la política de comercio interior. Horror vacuii y liberalización». Información Comercial Española, septiembre-octubre, 868, 17-29.

[4] CASARES, J.; ARANDA, E.; MARTÍN, V. J. y CASARES ARAÚZ DE ROBLES, J. (2013). Distribución comercial. Navarra: Aranzadi, cuarta edición.

[5] CASARES, J. y MARTÍN, V. J. (2015). «Convergencia de formatos comerciales». Distribución y Consumo, 136, 5-17.

[6] COMISIÓN EUROPEA (1999). Libro Blanco del Comercio. Comisión Europea, Bruselas.

[7] COMISIÓN EUROPEA (2010). Hacia un mercado interior del comercio y de la distribución más justo y eficaz en la perspectiva de 2020, Bruselas, 5.7.2010 COM (2010) 355 final.

[8] COMISIÓN EUROPEA (2010). EUROPA 2020. Una estrategia para un crecimiento inteligente, sostenible e integrador, Bruselas, 3.3.2010 COM (2010) 2020 final.

[9] COMISIÓN EUROPEA (2011). Primer Semestre Europeo de coordinación de las políticas económicas: Orientación de las políticas nacionales en 2011-2012, Bruselas, 7.6.2011 COM (2011) 400 final.

[10] COMISIÓN EUROPEA (2012). Un nuevo concepto de educación: invertir en las competencias para lograr mejores resultados socioeconómicos, Bruselas, 20.11.2012 COM (2012) 669 final.

[11] COMISIÓN EUROPEA (2012). Medidas en favor de la Estabilidad, el Crecimiento y el Empleo, Bruselas, 30.5.2012 COM (2012) 299 final.

[12] COMISIÓN EUROPEA (2013). Libro Verde sobre las prácticas comerciales desleales en la cadena de suministro alimentario y no alimentario entre empresas en Europa, Bruselas, 31.1.2013 COM (2013) 37 final.

[13] COMISIÓN EUROPEA (2013). Plan de Acción Europeo para el comercio minorista, Bruselas, 31.1.2013 COM (2013) 36 final.

[14] COMISIÓN EUROPEA (2013). Semestre Europeo 2013: recomendaciones específicas por país. Sacar a Europa de la crisis, Bruselas, 29.5.2013 COM (2013) 350 final.

[15] COMISIÓN EUROPEA (2014). Study on the legal framework covering business-to-business unfair trading practices in the retail supply chain, Final Report.http://ec.europa.eu/internal_market/retail/docs/140711-study-utp-legal-framework_en.pdf.

[16] COMISIÓNEUROPEA(2014). The economic impact of modern retail on choice and innovation in the EU food sector. Final Report. http://ec.europa.eu/competition/publications/KD0214955ENN. $p d f$.

[17] COMISIÓN EUROPEA (2014). Hacer frente a las prácticas comerciales desleales en la cadena de suministro alimentario entre empresas, Estrasburgo, 15.7.2014, COM (2014) 472 final.

[18] COMISIÓN EUROPEA (2014). Semestre Europeo 2014: recomendaciones específicas por país. Cimentar el crecimiento, Bruselas, 2.6.2014 COM (2014) 400 final.

[19] COMISIÓN EUROPEA (2015). Report of the High Level Group on Retail Competitiveness. http://ec.europa.eu/growth/single-market/services/retail/index_en.htm. 
[20] COMISIÓN EUROPEA (2015). Semestre Europeo 2015: recomendaciones específicas por país, Bruselas, 13.5.2015 COM (2015) 250 final.

[21] COMISIÓN EUROPEA (2016). Semestre Europeo 2016: recomendaciones específicas por país, Bruselas, 18.5.2016 COM (2016) 321 final.

[22] EUROSTAT (2016). Database. http://ec.europa.eu/eurostat/data/database.

[23] PARLAMENTO EUROPEO (2011). Resolución del Parlamento Europeo, de 5 de julio de 2011, sobre un mercado minorista más eficaz y más justo, Estrasburgo (2010/2109(INI)). 